\title{
Article \\ On the Total Neighbor Sum Distinguishing Index of IC-Planar Graphs
}

\author{
Donghan Zhang, Chao Li * and Fugang Chao
}

check for updates

Citation: Zhang, D.; Li, C.; Chao, F. On the Total Neighbor Sum Distinguishing Index of IC-Planar Graphs. Symmetry 2021, 13, 1787. https://doi.org/10.3390/sym13101787

Academic Editor: Magdalena

Lemańska

Received: 26 August 2021

Accepted: 22 September 2021

Published: 26 September 2021

Publisher's Note: MDPI stays neutral with regard to jurisdictional claims in published maps and institutional affiliations.

Copyright: (c) 2021 by the authors. Licensee MDPI, Basel, Switzerland. This article is an open access article distributed under the terms and conditions of the Creative Commons Attribution (CC BY) license (https:/ / creativecommons.org/licenses/by/ $4.0 /)$.
School of Mathematics and Computer Application, Shangluo University, Shangluo 726000, China; zhang_dh@mail.nwpu.edu.cn (D.Z.); chaofugang@126.com (F.C.)

* Correspondence: 129001@slxy.edu.cn

Abstract: A proper total $k$-coloring $\phi$ of $G$ with $\sum_{z \in E_{G}(u) \cup\{u\}} \phi(z) \neq \sum_{z \in E_{G}(v) \cup\{v\}} \phi(z)$ for each $u v \in E(G)$ is called a total neighbor sum distinguishing $k$-coloring, where $E_{G}(u)=\{u v \mid u v \in E(G)\}$. Pilśniak and Woźniak conjectured that every graph with maximum degree $\Delta$ exists a total neighbor sum distinguishing $(\Delta+3)$-coloring. In this paper, we proved that any IC-planar graph with $\Delta \geq 12$ satisfies this conjecture, which improves the result of Song and $\mathrm{Xu}$.

Keywords: combinatorial nullstellensatz; IC-planar graphs; total neighbor sum distinguishing coloring; discharging

\section{Introduction}

Let $G=(V(G), E(G))$ be a simple graph. Set $T(G)=V(G) \cup E(G)$ and $E_{G}(u)=$ $\{u v \mid u v \in E(G)\}$. A total neighbor sum distinguishing $k$-coloring (for short, $t n s d$ ) is a mapping $\phi: T(G) \rightarrow\{1,2, \cdots, k\}$ satisfying the following conditions:

(i) $\phi\left(z_{1}\right) \neq \phi\left(z_{2}\right)$ for any two adjacent or incident elements $z_{1}, z_{2}$ in $T(G)$;

(ii) $\sum_{z \in E_{G}(u) \cup\{u\}} \phi(z) \neq \sum_{z \in E_{G}(v) \cup\{v\}} \phi(z)$ for each $u v \in E(G)$.

The total neighbor sum distinguishing index of $G$, denoted by $\chi_{\Sigma}^{\prime \prime}(G)$, is the smallest integer $k$ such that $G$ has a tnsd $k$-coloring.

In 2015, Pilśniak and Woźniak [1] first studied the coloring and gave the conjecture about the tnsd index as follows:

Conjecture 1 ([1]). For every graph $G, \chi_{\Sigma}^{\prime \prime}(G) \leq \Delta(G)+3$.

Up to now, many special classes of graphs had been proved to satisfy Conjecture 1 , including complete graphs, bipartite graphs, subcubic graphs [1], 2-degenerate graphs with maximum degree $\Delta \geq 6$ [2], $K_{4}$-minor-free graphs [3], planar graphs with maximum degree $\Delta \geq 10$ [4], and planar graphs with maximum degree $\Delta \geq 7$ but without triangles [5].

A graph is 1-planar if it can be embedded on the plane so that each edge is crossed at most once. In 1965, Ringel introduced the notion of 1-planar graphs to color a planar graph and its dual simultaneously. In particularly, a 1-planar graph such that each vertex is incident with, at most, a crossing is called an IC-planar (independent crossing planar) graph, which was introduced by Albertson [6] while considering the relationship between the crossing number and the chromatic number.

Recently, the tnsd index on IC-planar graphs has been extensively studied. There are some recent results in the following.

Theorem 2. Each of the following classes of graphs satisfies Conjecture 1.

(1) IC-planar graph with $\Delta \geq 7$ but without triangles ([7]).

(2) IC-planar graph with $\Delta \geq 14$ but without 2-vertex incident with crossed edge ([8]).

(3) IC-planar graph with $\Delta \geq 13$ ([9]). 
There is also a result of list version about the tnsd index of IC-planar graphs, see [10].

In this paper, we discuss any IC-planar graph with maximum degree $\Delta \geq 12$ and obtain the following result, which extends the result of (3) in Theorem 2.

Theorem 3. Let $G$ be an IC-planar graph. Then, $\chi_{\Sigma}^{\prime \prime}(G) \leq \max \{\Delta(G)+3,15\}$.

\section{Preliminaries}

Let $G$ be a simple graph and $u v$ be an edge of $G$. For a vertex $u \in V(G)$, we use $d_{G}(u)$ and $N_{G}(u)$ to denote the degree and neighbors of $u$ in $G$, respectively. If $d_{G}(u)=\ell$ (resp., $d_{G}(u) \geq \ell, d_{G}(u) \leq \ell$ ), we call $u$ an $\ell$-vertex (resp., $\ell^{+}$-vertex, $\ell^{-}$-vertex) and an $\ell$-neighbor (resp., $\ell^{+}$-neighbor, $\ell^{-}$-neighbor) of $v$. We use $n_{G}^{\ell}(u)$ (resp., $n_{G}^{\ell^{+}}(u), n_{G}^{\ell^{-}}(u)$ ) to represent the number of $\ell$-vertices (resp., $\ell^{+}$-vertices, $\ell^{-}$-vertices) adjacent to $u$ in $G$. For two subsets $A$ and $B$ of $V(G)$, we denote by $[A, B]$ the set of edges with one end in $A$ and the other in $B$. In particular, for $u \in V(G)$, we write $[u, B]$ for $[\{u\}, B]$.

Theorem 4 ([11]). Assume that $\mathbb{F}$ is an arbitrary field and $P \in \mathbb{F}\left[x_{1}, \ldots, x_{n}\right]$ with degree $\operatorname{deg}(P)=\sum_{k=1}^{n} i_{k}$, where $i_{k} \geq 0$ is an integer. If the coefficient $c_{P}\left(x_{1}^{i_{1}}, \cdots, x_{n}^{i_{n}}\right)$ of the monomial $x_{1}^{i_{1}} \ldots x_{n}^{i_{n}}$ in $P$ is nonzero, and if $S_{1}, \ldots, S_{n}$ are subsets of $\mathbb{F}$ with $\left|S_{k}\right|>i_{k}$, there are $s_{1} \in S_{1}, \ldots, s_{n} \in S_{n}$ such that $P\left(s_{1}, \ldots, s_{n}\right) \neq 0$.

The planar graph $G^{\times}$obtained from a 1-planar graph $G$ by turning all crossings of $G$ into new 4-vertices on the plane is called the associated planar graph of $G$. Let $u v \in E\left(G^{\times}\right)$. Then, $u$ is called a false vertex and a false neighbor of $v$ if $u \in V\left(G^{\times}\right) \backslash V(G)$, and a real vertex and a real neighbor of $v$ otherwise. We use $F\left(G^{\times}\right)$to denote the set of faces in $G^{\times}$. For each face $f \in F\left(G^{\times}\right)$, we call $f$ a false face if it is incident with one false vertex, and a real face otherwise. A face with degree $\ell$ is called an $\ell$-face. Unless stated otherwise, an $\ell$-face means a real or false $\ell$-face throughout each section in the following.

Lemma 1 ([12]). Assume that $G$ is a 1-planar graph and $G^{\times}$is its associated planar graph. If a real 3-vertex $u$ is adjacent to a false vertex $v$ in $G^{\times}$, then $u v$ is not incident with two 3-faces in $G^{\times}$.

\section{Proof of Theorem 3}

Let $V_{i}(G)\left(V_{i^{-}}(G), V_{i^{+}}(G)\right.$, respectively) represent the set of vertices in $G$ of degree $i$ (at most $i$, at least $i$, respectively). For the graph $G$, a graph $G^{\prime}$ is smaller than it if one of the below conditions is satisfied.

(i) $\left|E\left(G^{\prime}\right)\right|<|E(G)|$;

(ii) In the lexicographic order, $\left(\left|V_{t}\left(G^{\prime}\right)\right|,\left|V_{t-1}\left(G^{\prime}\right)\right|, \cdots,\left|V_{1}\left(G^{\prime}\right)\right|\right)$ precedes $\left(\left|V_{t}(G)\right|\right.$, $\left.\left|V_{t-1}(G)\right|, \cdots,\left|V_{1}(G)\right|\right)$, where $t=\max \left\{\Delta\left(G^{\prime}\right), \Delta(G)\right\}$.

We say a graph is minimal for some property if no smaller graph admits the property. To obtain a smaller graph, $G^{\prime}$, than $G$, we will frequently delete some edges or split some vertices of $G$ in the following.

From now on, let $G$ be a minimal graph not satisfying Theorem 3 and $k=\max \{\Delta(G)+$ $3,15\}$. Then, any graph $G^{\prime}$ that is smaller than $G$ has a tnsd $k$-coloring $\phi^{\prime}$. By splitting a vertex $v$ into two vertices $v_{1}$ and $v_{2}$, we obtain a smaller graph $G^{\prime}$ than $G$, then $G^{\prime}$ has a tnsd $k$-coloring $\phi^{\prime}$. If we can obtain a tnsd $k$-coloring of $G$ from the $\phi^{\prime}$ by sticking $v_{1}$ and $v_{2}$ into $v$, we say that $v_{1}$ and $v_{2}$ can be properly stunk. In the following, we will often extend a tnsd $k$-coloring $\phi^{\prime}$ of $G^{\prime}$ to a tnsd $k$-coloring $\phi$ of $G$ to obtain a contradiction. Set $m_{\phi}(u)=\sum_{z \in E_{G}(u) \cup\{u\}} \phi(z)$. Assume that $X$ is a subset of $T(G)$ and $\psi: X \rightarrow\{1, \cdots, k\}$ is a mapping. For each $z \in T(G)$, set

$$
S(z)=\{1, \cdots, k\} \backslash\{\psi(x) \mid x \text { is adjacent or incident to } z \text { in } G\} .
$$


Assume that $u$ is a $4^{-}$-vertex in $G$ and $\phi^{\prime}: T(G) \backslash\{u\} \rightarrow\{1, \cdots, k\}$ is a mapping satisfying the conditions as follows:

(i) $\phi^{\prime}\left(z_{1}\right) \neq \phi^{\prime}\left(z_{2}\right)$ for any adjacent or incident elements $z_{1}, z_{2} \in T(G) \backslash\{u\}$;

(ii) $\sum_{z \in E_{G}\left(z_{1}\right) \cup\left\{z_{1}\right\}} \phi^{\prime}(z) \neq \sum_{z \in E_{G}\left(z_{2}\right) \cup\left\{z_{2}\right\}} \phi^{\prime}(z)$ for any two adjacent vertices $z_{1}, z_{2} \in$ $V(G) \backslash\{u\}$.

Since $S(u)=\{1, \cdots, k\} \backslash\left\{\phi^{\prime}(x) \mid x\right.$ is adjacent or incident to $u$ in $\left.G\right\},|S(u)| \geq k-$ $2 d_{G}(u)>d_{G}(u)$. Thus, we can extend the $\phi^{\prime}$ to a tnsd $k$-coloring $\phi$ of $G$, which is a contradiction. For simplicity, we will omit the colors of all $4^{-}$-vertices in the following discussion.

In Figures 1 and 2, a black vertex represents that its neighbors are all depicted and a white vertex means that its neighbors may not be all depicted.

Claim 1. The graph $G$ contains no configuration in Figure 1.

Proof. Suppose that $G$ contains the configuration in Figure 1. By splitting the two 3-vertices $v_{2}, v_{4}$ of the configuration in Figure 1 into $v_{21}, v_{22}$ and $v_{41}, v_{42}$, respectively, we obtain a smaller graph $G^{\prime}$ than $G$. Then, $G^{\prime}$ has a tnsd $k$-coloring $\phi^{\prime}$. Without loss of generality, set $\phi^{\prime}\left(v v_{1}\right)=c_{1}, \phi^{\prime}\left(v v_{22}\right)=c_{2}, \phi^{\prime}\left(v v_{3}\right)=c_{3}, \phi^{\prime}\left(v v_{42}\right)=c_{4}, \phi^{\prime}\left(v v_{5}\right)=c_{5}, \phi^{\prime}\left(v_{1} v_{21}\right)=c_{6}$, $\phi^{\prime}\left(v_{21} v_{3}\right)=c_{7}, \phi^{\prime}\left(v_{3} v_{41}\right)=c_{8}$, and $\phi^{\prime}\left(v_{41} v_{5}\right)=c_{9}$ (see Figure 2).

If we can properly stick $v_{21}, v_{41}$ with $v_{22}, v_{42}$, respectively, we can obtain a tnsd $k$ coloring $\phi$ of $G$, which is a contradiction. Thus, assume that at least one of $v_{21}$ and $v_{41}$ cannot be stuck properly below. Let $Y=T\left(G^{\prime}\right) \backslash\left\{v_{21}, v_{22}, v_{41}, v_{42}\right\}$.

Case 1. Suppose that one of $v_{21}$ and $v_{41}$ cannot be stuck properly. If $v_{21}$ can not be stuck properly, $c_{2} \in\left\{c_{6}, c_{7}\right\}$. Without loss of generosity, set $c_{2}=c_{6}$.

Set $c_{8} \neq c_{6}, c_{9} \neq c_{6}$, and $c_{4} \neq c_{7}$. Then, we assign $\phi^{\prime \prime}\left(v v_{22}\right)=c_{4}, \phi^{\prime \prime}\left(v v_{42}\right)=c_{2}$, and $\phi(z)^{\prime \prime}=\phi^{\prime}(z)$ for every $z \in Y \backslash\left\{v v_{22}, v v_{42}\right\}$.

Set $c_{8} \neq c_{6}, c_{9} \neq c_{6}$, and $c_{4}=c_{7}$. Then, we assign $\phi^{\prime \prime}\left(v v_{22}\right)=c_{4}, \phi^{\prime \prime}\left(v v_{42}\right)=c_{2}$, $\phi^{\prime \prime}\left(v_{21} v_{3}\right)=c_{8}, \phi^{\prime \prime}\left(v_{3} v_{41}\right)=c_{7}$, and $\phi(z)^{\prime \prime}=\phi^{\prime}(z)$ for every $z \in Y \backslash\left\{v v_{22}, v v_{42}, v_{21} v_{3}, v_{3} v_{41}\right\}$.

Set $c_{8}=c_{6}$. If $c_{9} \neq c_{3}$, we assign $\phi^{\prime \prime}\left(v v_{22}\right)=c_{3}, \phi^{\prime \prime}\left(v v_{3}\right)=c_{2}, \phi^{\prime \prime}\left(v_{3} v_{41}\right)=c_{3}$, and $\phi(z)^{\prime \prime}=\phi^{\prime}(z)$ for every $z \in Y \backslash\left\{v v_{22}, v v_{3}, v_{3} v_{41}\right\}$. If $c_{9}=c_{3}$ and $c_{5} \neq c_{7}$, we assign $\phi^{\prime \prime}\left(v v_{22}\right)=c_{5}, \phi^{\prime \prime}\left(v v_{3}\right)=c_{8}, \phi^{\prime \prime}\left(v_{3} v_{41}\right)=c_{3}, \phi^{\prime \prime}\left(v v_{5}\right)=c_{9}, \phi^{\prime \prime}\left(v_{41} v_{5}\right)=c_{5}$, and $\phi(z)^{\prime \prime}=\phi^{\prime}(z)$ for every $z \in Y \backslash\left\{v v_{22}, v v_{3}, v_{3} v_{41}, v v_{5}, v_{41} v_{5}\right\}$. If $c_{9}=c_{3}$ and $c_{5}=c_{7}$, we assign $\phi^{\prime \prime}\left(v v_{1}\right)=c_{6}, \phi^{\prime \prime}\left(v_{1} v_{21}\right)=c_{1}, \phi^{\prime \prime}\left(v v_{22}\right)=c_{4}, \phi^{\prime \prime}\left(v v_{42}\right)=c_{1}$, and $\phi(z)^{\prime \prime}=\phi^{\prime}(z)$ for every $z \in Y \backslash\left\{v v_{1}, v_{1} v_{21}, v v_{22}, v v_{42}\right\}$.

Set $c_{9}=c_{6}$. If $c_{8} \neq c_{5}$ and $c_{5} \neq c_{7}$, we assign $\phi^{\prime \prime}\left(v v_{22}\right)=c_{5}, \phi^{\prime \prime}\left(v v_{5}\right)=c_{2}$, $\phi^{\prime \prime}\left(v_{41} v_{5}\right)=c_{5}$, and $\phi(z)^{\prime \prime}=\phi^{\prime}(z)$ for every $z \in Y \backslash\left\{v v_{22}, v v_{5}, v_{41} v_{5}\right\}$. If $c_{8} \neq c_{5}$ and $c_{5}=$ $c_{7}$, we assign $\phi^{\prime \prime}\left(v v_{22}\right)=c_{4}, \phi^{\prime \prime}\left(v v_{5}\right)=c_{9}, \phi^{\prime \prime}\left(v_{41} v_{5}\right)=c_{5}, \phi^{\prime \prime}\left(v v_{3}\right)=c_{7}, \phi^{\prime \prime}\left(v_{21} v_{3}\right)=c_{3}$, $\phi^{\prime \prime}\left(v v_{42}\right)=c_{3}$, and $\phi(z)^{\prime \prime}=\phi^{\prime}(z)$ for every $z \in Y \backslash\left\{v v_{22}, v_{21} v_{3}, v v_{3}, v v_{42}, v v_{5}, v_{41} v_{5}\right\}$. If $c_{8}=c_{5}$, we assign $\phi^{\prime \prime}\left(v v_{22}\right)=c_{3}, \phi^{\prime \prime}\left(v v_{5}\right)=c_{9}, \phi^{\prime \prime}\left(v_{41} v_{5}\right)=c_{5}, \phi^{\prime \prime}\left(v v_{3}\right)=c_{8}$, $\phi^{\prime \prime}\left(v_{3} v_{41}\right)=c_{3}$, and $\phi(z)^{\prime \prime}=\phi^{\prime}(z)$ for every $z \in Y \backslash\left\{v v_{22}, v v_{3}, v_{3} v_{41}, v v_{5}, v_{41} v_{5}\right\}$.

In each case above, by sticking $v_{i 1}$ and $v_{i 2}$ into $v_{i}$ for $i=2,4$, we obtain the graph $G$. Since both $v_{2}$ and $v_{4}$ are 3 -vertices in $G$, we can obtain a tnsd $k$-coloring $\phi$ of $G$ from $\phi^{\prime \prime}$. By symmetry, if $v_{41}$ cannot be stuck properly, we can also obtain a tnsd $k$-coloring $\phi$ of $G$. It is a contradiction.

Case 2. Assume that both $v_{21}$ and $v_{41}$ cannot be stuck properly. Then, $c_{2}=c_{6}=c_{8}$ and $c_{4}=c_{7}=c_{9}$. Let $\phi^{\prime \prime}\left(v v_{1}\right)=c_{6}, \phi^{\prime \prime}\left(v_{1} v_{21}\right)=c_{1}, \phi^{\prime \prime}\left(v v_{5}\right)=c_{9}, \phi^{\prime \prime}\left(v_{41} v_{5}\right)=c_{5}, \phi^{\prime \prime}\left(v v_{22}\right)=$ $c_{5}, \phi^{\prime \prime}\left(v v_{42}\right)=c_{1}$, and $\phi(z)^{\prime \prime}=\phi^{\prime}(z)$ for every $z \in Y \backslash\left\{v v_{1}, v_{1} v_{21}, v v_{5}, v_{41} v_{5}, v v_{22}, v v_{42}\right\}$. By sticking $v_{i 1}$ and $v_{i 2}$ into $v_{i}$ for $i=2,4$, we obtain the graph $G$. Since both $v_{2}$ and $v_{4}$ are 3 -vertices in $G$, we can obtain a tnsd $k$-coloring $\phi$ of $G$ from $\phi^{\prime \prime}$, which is a contradiction. 


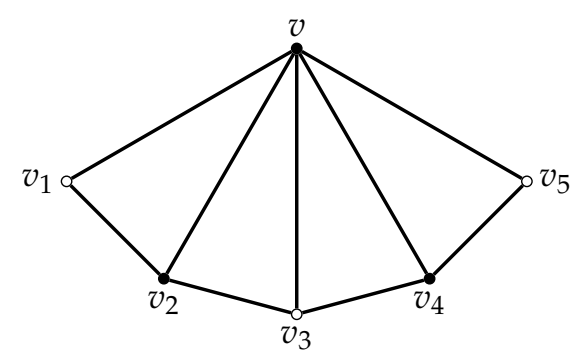

Figure 1. No configuration in $G$.

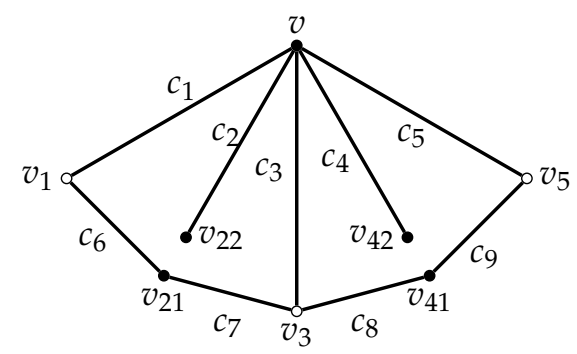

Figure 2. No configuration with colors in $G$.

The below Claim 2 follows from (3) in Theorem 2.

Claim 2. $\Delta(G) \leq 12$.

Claim 3. If $u \in V_{6^{-}}(G)$, then $\left|\left[u, V_{5^{-}}(G)\right]\right|=0$.

Proof. By contradiction, suppose that there is a vertex $u \in V_{6^{-}}(G)$ and a vertex $v \in V_{5^{-}}(G)$ such that $u v \in\left[u, V_{5^{-}}(G)\right]$. Let $G^{\prime}=G-u v$. Then, $G^{\prime}$ has a tnsd $k$-coloring $\phi^{\prime}$. We first erase the colors on $u$ and $v$ from $\phi^{\prime}$. Without loss of generality, set $d_{G}(u)=6$ and $d_{G}(v)=5$. Then,

$$
\begin{aligned}
|S(u)| & \geq k-2 \times(6-1) \geq 5 \\
|S(u v)| & \geq k-(6-1)-(5-1) \geq 6 \text { and } \\
|S(v)| & \geq k-2 \times(5-1) \geq 7 .
\end{aligned}
$$

Let $\phi$ be a map on $T(G)$ obtained from $\phi^{\prime}$ by assigning $\phi(u)=x_{1}, \phi(v)=x_{2}, \phi(u v)=$ $y_{1}$, and $\phi(z)=\phi^{\prime}(z)$ for every $z \in T(G) \backslash\{u, v, u v\}$. Set

$$
\begin{aligned}
P\left(x_{1}, x_{2}, y_{1}\right)= & \prod_{z \in N_{G}(u) \backslash\{v\}}\left(m_{\phi}(u)-m_{\phi}(z)\right) \prod_{z \in N_{G}(v) \backslash\{u\}}\left(m_{\phi}(v)-m_{\phi}(z)\right) \\
& \cdot\left(m_{\phi}(u)-m_{\phi}(v)\right)\left(x_{1}-x_{2}\right)\left(x_{1}-y_{1}\right)\left(x_{2}-y_{1}\right) .
\end{aligned}
$$

With the help of MATHEMATICA, we have $c_{P}\left(x_{1}^{4} x_{2}^{4} y_{1}^{5}\right)=-25$. By Theorem 4 , there is a color $c_{1} \in S(u)$, a color $c_{2} \in S(v)$, and a color $c_{3} \in S(u v)$ such that $P\left(c_{1}, c_{2}, c_{3}\right) \neq 0$. Therefore, we can extend the $\phi^{\prime}$ to a tnsd $k$-coloring $\phi$ of $G$ by recoloring $u, v$ with colors $c_{1}, c_{2}$ and coloring $u v$ with color $c_{3}$, respectively. It is a contradiction.

Claim 4. If $u \in V_{7}(G),\left|\left[u, V_{4^{-}}(G)\right]\right|=0$ and $\left|\left[u, V_{5}(G)\right]\right| \leq 1$.

Proof. We first prove $\left|\left[u, V_{4^{-}}(G)\right]\right|=0$ when $u \in V_{7}(G)$. By contradiction, suppose that there is a vertex $u \in V_{7}(G)$ and a vertex $v \in V_{4^{-}}(G)$ such that $u v \in\left[u, V_{4^{-}}(G)\right]$. Let 
$G^{\prime}=G-u v$. Then, $G^{\prime}$ has a tnsd $k$-coloring $\phi^{\prime}$. We first erase the colors on $u$ and $v$ from $\phi^{\prime}$. Note that $v$ is a $4^{-}$-vertex. Then,

$$
\begin{gathered}
|S(u)| \geq k-2 \times(7-1) \geq 3 \text { and } \\
|S(u v)| \geq k-(7-1)-(4-1) \geq 6 .
\end{gathered}
$$

Let $\phi$ be a map on $T(G) \backslash\{v\}$ obtained from $\phi^{\prime}$ by assigning $\phi(u)=x_{1}, \phi(u v)=y_{1}$, and $\phi(z)=\phi^{\prime}(z)$ for every $z \in T(G) \backslash\{u, v, u v\}$. Set

$$
P\left(x_{1}, y_{1}\right)=\prod_{z \in N_{G}(u) \backslash\{v\}}\left(m_{\phi}(u)-m_{\phi}(z)\right)\left(x_{1}-y_{1}\right) .
$$

By applying MATHEMATICA, we have $c_{P}\left(x_{1}^{2} y_{1}^{5}\right)=-9$. By Theorem 4 , there is a color $c_{1} \in S(u)$ and a color $c_{2} \in S(u v)$ such that $P\left(c_{1}, c_{2}\right) \neq 0$.

Since $v$ is a $4^{-}$-vertex, we can obtain a tnsd $k$-coloring of $G$ from the $\phi^{\prime}$ by recoloring $u$ with color $c_{1}$ and coloring $u v$ with color $c_{2}$, respectively. This is a contradiction.

Next, we show $\left|\left[u, V_{5}(G)\right]\right| \leq 1$ when $u \in V_{7}(G)$. Suppose, to the contrary, that there is a vertex $u \in V_{7}(G)$ and two vertices $v_{1}, v_{2} \in V_{5}(G)$ such that $u v_{1}, u v_{2} \in\left[u, V_{5}(G)\right]$. Let $G^{\prime}=G-u v_{1}-u v_{2}$. Then, $G^{\prime}$ has a tnsd $k$-coloring $\phi^{\prime}$. We first erase the colors on $u, v_{1}$, and $v_{2}$ from $\phi^{\prime}$. Then,

$$
\begin{aligned}
|S(u)| & \geq k-2 \times(7-2) \geq 5, \\
\left|S\left(u v_{i}\right)\right| & \geq k-(7-2)-(5-1) \geq 6 \text { and } \\
\left|S\left(v_{i}\right)\right| & \geq k-2 \times(5-1) \geq 7 \text { for } i=1,2 .
\end{aligned}
$$

Let $\phi$ be a map on $T(G)$ obtained from $\phi^{\prime}$ by assigning $\phi(u)=x_{1}, \phi\left(v_{i}\right)=x_{i+1}$, and $\phi\left(u v_{i}\right)=y_{i}$ for $i=1,2$, and $\phi(z)=\phi^{\prime}(z)$ for every $z \in T(G) \backslash\left\{u, v_{1}, v_{2}, u v_{1}, u v_{2}\right\}$. Set

$$
\begin{aligned}
P\left(x_{1}, x_{2}, x_{3}, y_{1}, y_{2}\right)= & \prod_{z \in N_{G}(u) \backslash\left\{v_{1}, v_{2}\right\}}\left(m_{\phi}(u)-m_{\phi}(z)\right) \prod_{i=1}^{2}\left(m_{\phi}(u)-m_{\phi}\left(v_{i}\right)\right) \\
& \cdot \prod_{i=1}^{2} \prod_{z \in N_{G}\left(v_{i}\right) \backslash\{u\}}\left(m_{\phi}\left(v_{i}\right)-m_{\phi}(z)\right) \prod_{i=1}^{2}\left(x_{1}-x_{i+1}\right) \\
& \cdot\left(y_{1}-y_{2}\right) \prod_{i=1}^{2}\left(x_{1}-y_{i}\right)\left(x_{i+1}-y_{i}\right) .
\end{aligned}
$$

With the help of MATHEMATICA, we have $c_{P}\left(x_{1}^{4} x_{2}^{4} x_{3}^{5} y_{1}^{4} y_{2}^{5}\right)=120$. By Theorem 4 , there is a color $c_{1} \in S(u)$, a color $c_{i+1} \in S\left(v_{i}\right)$, and a color $c_{i+3} \in S\left(u v_{i}\right)$ for $i=1,2$ such that $P\left(c_{1}, \cdots, c_{5}\right) \neq 0$. Therefore, we can extend the $\phi^{\prime}$ to a tnsd $k$-coloring $\phi$ of $G$ by recoloring $u, v_{i}$ with colors $c_{1}, c_{i+1}$ and coloring $u v_{i}$ with color $c_{i+3}$ for $i=1,2$, respectively. This is a contradiction.

Claim 5. If $u \in V_{\ell}(G)$ with $8 \leq \ell \leq 9$, then,

(1) $\left|\left[u, V_{4^{-}}(G)\right]\right| \leq \ell-7$,

(2) $\left|\left[u, V_{5^{-}}(G)\right]\right| \leq \ell-7$ when $\left|\left[u, V_{4^{-}}(G)\right]\right| \geq 1$.

Proof. Suppose to be contrary that there is a vertex $u \in V_{\ell}(G)$, a vertex $v_{1} \in V_{4^{-}}(G)$, and $(\ell-7)$ vertices $v_{2}, \cdots, v_{\ell-6} \in V_{5^{-}}(G)$ with $8 \leq \ell \leq 9$ such that $u v_{1} \in\left[u, V_{4^{-}}(G)\right]$ and $u v_{i} \in\left[u, V_{5^{-}}(G)\right]$ for $i=2, \cdots, \ell-6$. Let $G^{\prime}=G-\left\{u v_{i} \mid i=1, \cdots, \ell-6\right\}$. Then, $G^{\prime}$ has a 
tnsd $k$-coloring $\phi^{\prime}$. We first erase the colors on $u$ and $v_{i}$ with $1 \leq i \leq \ell-6$ from $\phi^{\prime}$. Without loss of generality, set $d_{G}\left(v_{i}\right)=5$ with $2 \leq i \leq \ell-6$. Note that $v_{1}$ is a $4^{-}$-vertex. Then,

$$
\begin{aligned}
|S(u)| & \geq k-2 \times(\ell-(\ell-6)) \geq 3, \\
\left|S\left(u v_{1}\right)\right| & \geq k-(\ell-(\ell-6))-(4-1) \geq 6, \\
\left|S\left(u v_{i}\right)\right| & \geq k-(\ell-(\ell-6))-(5-1) \geq 5 \text { and } \\
\left|S\left(v_{i}\right)\right| & \geq k-2 \times(5-1) \geq 7 \text { for } i=2, \cdots, \ell-6 .
\end{aligned}
$$

Let $\phi$ be a map on $T(G) \backslash\left\{v_{1}\right\}$ obtained from $\phi^{\prime}$ by assigning

$$
\phi(u)=x_{1}, \phi\left(v_{2}\right)=x_{2}, \cdots, \phi\left(v_{\ell-6}\right)=x_{\ell-6}, \phi\left(u v_{1}\right)=y_{1}, \cdots, \phi\left(u v_{\ell-6}\right)=y_{\ell-6}
$$

and $\phi(z)=\phi^{\prime}(z)$ for every $z \in T(G) \backslash\left\{u, v_{1}, \cdots, v_{\ell-6}, u v_{1}, \cdots, u v_{\ell-6}\right\}$. Set

$$
\begin{aligned}
P\left(x_{1}, \cdots, x_{\ell-6}, y_{1}, \cdots, y_{\ell-6}\right)= & \prod_{z \in N_{G}(u) \backslash\left\{v_{1}, \cdots, v_{\ell-6}\right\}}\left(m_{\phi}(u)-m_{\phi}(z)\right) \prod_{i=2}^{\ell-6}\left(m_{\phi}(u)-m_{\phi}\left(v_{i}\right)\right) \\
& \cdot \prod_{i=2}^{\ell-6} \prod_{z \in N_{G}\left(v_{i}\right) \backslash\{u\}}\left(m_{\phi}\left(v_{i}\right)-m_{\phi}(z)\right) \prod_{i=2}^{\ell-6}\left(x_{1}-x_{i}\right)\left(x_{i}-y_{i}\right) \\
& \cdot \prod_{i=1}^{\ell-6}\left(x_{1}-y_{i}\right) \prod_{1 \leq i<j \leq \ell-6}\left(y_{i}-y_{j}\right) .
\end{aligned}
$$

When $\ell=8, \ell-6=2$, and we have $c_{P}\left(x_{1}^{2} x_{2}^{6} y_{1}^{4} y_{2}^{4}\right)=20$ by applying MATHEMATICA. When $\ell=9, \ell-6=3$, and we have $c_{P}\left(x_{1}^{2} x_{2}^{6} x_{3}^{5} y_{1}^{5} y_{2}^{4} y_{2}^{4}\right)=-59$ by applying MATHEMATICA. By Theorem 4, there is a color $c_{1} \in S(u)$, a color $c_{i} \in S\left(v_{i}\right)$ with $2 \leq i \leq \ell-6$, and a color $c_{i+\ell-6} \in S\left(u v_{i}\right)$ with $1 \leq i \leq \ell-6$ such that $P\left(c_{1}, \cdots, c_{2 \ell-12}\right) \neq 0$ when $8 \leq \ell \leq 9$. Since $v_{1}$ is a $4^{-}$-vertex, we can extend the $\phi^{\prime}$ to a tnsd $k$-coloring of $G$ by recoloring $u, v_{2}, \cdots, v_{\ell-6}$ with colors $c_{1}, \cdots, c_{\ell-6}$ and coloring $u v_{1}, \cdots, u v_{\ell-6}$ with colors $c_{\ell-5}, \cdots, c_{2 \ell-12}$, respectively. This is a contradiction.

Claim 6. If $u \in V_{\ell}(G)$ with $10 \leq \ell \leq 11$, then

(1) $\left|\left[u, V_{3^{-}}(G)\right]\right| \leq \ell-7$,

(2) $\left|\left[u, V_{4^{-}}(G)\right]\right| \leq \ell-7$ when $\left|\left[u, V_{3^{-}}(G)\right]\right| \geq 1$,

(3) $\left|\left[u, V_{5}(G)\right]\right|=0$ when $\left|\left[u, V_{3^{-}}(G)\right]\right| \geq 1$ and $\left|\left[u, V_{4^{-}}(G)\right]\right|=\ell-7$.

Proof. By contradiction, suppose that there is a vertex $u \in V_{\ell}(G)$, a vertex $v_{1} \in V_{5^{-}}(G)$, a vertex $v_{2} \in V_{3^{-}}(G),(\ell-8)$ vertices $v_{3}, \cdots, v_{\ell-6} \in V_{4^{-}}(G)$ with $10 \leq \ell \leq 11$ such that $u v_{1} \in\left[u, V_{5^{-}}(G)\right], u v_{2} \in\left[u, V_{3^{-}}(G)\right]$, and $u v_{i} \in\left[u, V_{4^{-}}(G)\right]$ for $i=3, \cdots, \ell-6$. Let $G^{\prime}=G-\left\{u v_{i} \mid i=1, \cdots, \ell-6\right\}$. Then, $G^{\prime}$ has a tnsd $k$-coloring $\phi^{\prime}$. We first erase the colors on $u$ and $v_{i}$ with $1 \leq i \leq \ell-6$ from $\phi^{\prime}$. Without loss of generality, set $d_{G}\left(v_{1}\right)=5$. Note that $v_{2}$ is a $3^{-}$-vertex and $v_{i}$ is a $4^{-}$-vertex with $3 \leq i \leq \ell-6$. Then,

$$
\begin{aligned}
|S(u)| & \geq k-2 \times(\ell-(\ell-6)) \geq 3 \\
\left|S\left(v_{1}\right)\right| & \geq k-2 \times(5-1) \geq 7 \\
\left|S\left(u v_{1}\right)\right| & \geq k-(\ell-(\ell-6))-(5-1) \geq 5 \\
\left|S\left(u v_{2}\right)\right| & \geq k-(\ell-(\ell-6))-(3-1) \geq 7 \text { and } \\
\left|S\left(u v_{i}\right)\right| & \geq k-(\ell-(\ell-6))-(4-1) \geq 6 \text { for } i=3, \cdots, \ell-6 .
\end{aligned}
$$

Let $\phi$ be a map on $T(G) \backslash\left\{v_{2}, \cdots, v_{\ell-6}\right\}$ obtained from $\phi^{\prime}$ by assigning

$$
\phi(u)=x_{1}, \phi\left(v_{1}\right)=x_{2}, \phi\left(u v_{1}\right)=y_{1}, \cdots, \phi\left(u v_{\ell-6}\right)=y_{\ell-6}
$$


and $\phi(z)=\phi^{\prime}(z)$ for every $z \in T(G) \backslash\left\{u, v_{1}, \cdots, v_{\ell-6}, u v_{1}, \cdots, u v_{\ell-6}\right\}$. Set

$$
\begin{aligned}
P\left(x_{1}, x_{2}, y_{1}, \cdots, y_{\ell-6}\right)= & \prod_{z \in N_{G}(u) \backslash\left\{v_{1}, \cdots, v_{\ell-6}\right\}}\left(m_{\phi}(u)-m_{\phi}(z)\right) \prod_{z \in N_{G}\left(v_{1}\right) \backslash\{u\}}\left(m_{\phi}\left(v_{1}\right)-m_{\phi}(z)\right) \\
& \cdot\left(m_{\phi}(u)-m_{\phi}\left(v_{1}\right)\right)\left(x_{1}-x_{2}\right)\left(x_{2}-y_{1}\right) \\
& \cdot \prod_{i=1}^{\ell-6}\left(x_{1}-y_{i}\right) \prod_{1 \leq i<j \leq \ell-6}\left(y_{i}-y_{j}\right) .
\end{aligned}
$$

When $\ell=10, \ell-6=4$, and we have $c_{P}\left(x_{1}^{2} x_{2}^{6} y_{1}^{4} y_{2}^{6} y_{3}^{4} y_{4}\right)=-20$ with the help of MATHEMATICA. When $\ell=11, \ell-6=5$, and we have $c_{P}\left(x_{1}^{2} x_{2}^{6} y_{1}^{4} y_{2}^{6} y_{3}^{5} y_{4}^{4} y_{5}\right)=-4$ with the help of MATHEMATICA. By Theorem 4, there is a color $c_{1} \in S(u)$, a color $c_{2} \in S\left(v_{1}\right)$, and a color $c_{i+2} \in S\left(u v_{i}\right)$ with $1 \leq i \leq \ell-6$ such that $P\left(c_{1}, \cdots, c_{\ell-4}\right) \neq 0$ when $10 \leq \ell \leq 11$. Since $v_{i}$ is a $4^{-}$-vertex with $2 \leq i \leq \ell-6$, we can obtain a tnsd $k$-coloring of $G$ from the $\phi^{\prime}$ by recoloring $u, v_{1}$ with colors $c_{1}, c_{2}$ and coloring $u v_{1}, \cdots, u v_{\ell-6}$ with colors $c_{3}, \cdots, c_{\ell-4}$, respectively. This is a contradiction.

Claim 7. If $u \in V_{12}(G)$, then

(1) $\left|\left[u, V_{2^{-}}(G)\right]\right| \leq 5$,

(2) $\left|\left[u, V_{3^{-}}(G)\right]\right| \leq 5$ when $\left|\left[u, V_{2^{-}}(G)\right]\right| \geq 1$,

(3) $\left|\left[u, V_{4^{-}}(G)\right]\right| \leq 5$ when $\left|\left[u, V_{2^{-}}(G)\right]\right| \geq 1$ and $\left|\left[u, V_{3^{-}}(G)\right]\right| \geq 2$,

(4) $\left|\left[u, V_{5}(G)\right]\right|=0$ when $\left|\left[V_{2^{-}}(G), u\right]\right| \geq 1,\left|\left[V_{3^{-}}(G), u\right]\right| \geq 2$ and $\left|\left[u, V_{4^{-}}(G)\right]\right|=5$.

Proof. Suppose to the contrary that there is a vertex $u \in V_{12}(G)$, a vertex $v_{1} \in V_{5^{-}}(G)$, a vertex $v_{2} \in V_{2^{-}}(G)$, a vertex $v_{3} \in V_{3^{-}}(G)$, and three vertices $v_{4}, v_{5}, v_{6} \in V_{4^{-}}(G)$ such that $u v_{1} \in\left[u, V_{5^{-}}(G)\right], u v_{2} \in\left[u, V_{2^{-}}(G)\right], u v_{3} \in\left[u, V_{3^{-}}(G)\right]$, and $u v_{i} \in\left[u, V_{4^{-}}(G)\right]$ for $i=4,5,6$. Let $G^{\prime}=G-\left\{u v_{i} \mid i=1, \cdots, 6\right\}$. Then, $G^{\prime}$ has a tnsd $k$-coloring $\phi^{\prime}$. We first erase the colors on $u$ and $v_{i}$ with $1 \leq i \leq 6$ from $\phi^{\prime}$. Without loss of generality, set $d_{G}\left(v_{1}\right)=5$. Note that $v_{2}$ is a $2^{-}$-vertex, $v_{3}$ is a $3^{-}$-vertex, and $v_{i}$ is a $4^{-}$-vertex with $4 \leq i \leq 6$. Then,

$$
\begin{aligned}
|S(u)| & \geq k-2 \times(12-6) \geq 3 \\
\left|S\left(v_{1}\right)\right| & \geq k-2 \times(5-1) \geq 7 \\
\left|S\left(u v_{1}\right)\right| & \geq k-(12-6)-(5-1) \geq 5 \\
\left|S\left(u v_{2}\right)\right| & \geq k-(12-6)-(2-1) \geq 8 \\
\left|S\left(u v_{3}\right)\right| & \geq k-(12-6)-(3-1) \geq 7 \text { and } \\
\left|S\left(u v_{i}\right)\right| & \geq k-(12-6)-(4-1) \geq 6 \text { for } i=4,5,6 .
\end{aligned}
$$

Let $\phi$ be a map on $T(G) \backslash\left\{v_{2}, \cdots, v_{6}\right\}$ obtained from $\phi^{\prime}$ by assigning

$$
\phi(u)=x_{1}, \phi\left(v_{1}\right)=x_{2}, \phi\left(u v_{1}\right)=y_{1}, \cdots, \phi\left(u v_{6}\right)=y_{6}
$$

and $\phi(z)=\phi^{\prime}(z)$ for every $z \in T(G) \backslash\left\{u, v_{1}, \cdots, v_{6}, u v_{1}, \cdots, u v_{6}\right\}$. Set

$$
\begin{aligned}
P\left(x_{1}, x_{2}, y_{1}, \cdots, y_{6}\right)= & \prod_{z \in N_{G}(u) \backslash\left\{v_{1}, \cdots, v_{6}\right\}}\left(m_{\phi}(u)-m_{\phi}(z)\right) \prod_{z \in N_{G}\left(v_{1}\right) \backslash\{u\}}\left(m_{\phi}\left(v_{1}\right)-m_{\phi}(z)\right) \\
& \cdot\left(m_{\phi}(u)-m_{\phi}\left(v_{1}\right)\right)\left(x_{1}-x_{2}\right)\left(x_{2}-y_{1}\right) \\
& \cdot \prod_{i=1}^{6}\left(x_{1}-y_{i}\right) \prod_{1 \leq i<j \leq 6}\left(y_{i}-y_{j}\right) .
\end{aligned}
$$

With the help of MATHEMATICA, we have $c_{P}\left(x_{1}^{2} x_{2}^{6} y_{1}^{4} y_{2}^{7} y_{3}^{6} y_{4}^{5} y_{5}^{4}\right)=-4$. By Theorem 4 , there is a color $c_{1} \in S(u)$, a color $c_{2} \in S\left(v_{1}\right)$, and a color $c_{i+2} \in S\left(u v_{i}\right)$ with $1 \leq i \leq 6$ such that $P\left(c_{1}, \cdots, c_{8}\right) \neq 0$. Since $v_{i}$ is a $4^{-}$-vertex with $2 \leq i \leq 6$, we can obtain a tnsd 
$k$-coloring of $G$ from the $\phi^{\prime}$ by recoloring $u, v_{1}$ with colors $c_{1}, c_{2}$ and coloring $u v_{1}, \cdots, u v_{6}$ with colors $c_{3}, \cdots, c_{8}$, respectively. This is a contradiction.

Claim 8. Let $C_{3}=v_{1} v_{2} v_{3} v_{1}$ be a 3-cycle in $G$. Then, $\left(d_{G}\left(v_{1}\right), d_{G}\left(v_{2}\right), d_{G}\left(v_{3}\right)\right) \neq\left(6^{-}, 6^{-}, 7^{-}\right)$.

The following Equation (1) follows from Claims 3 7.

$$
\begin{cases}n_{G}^{5-}(u)=0 & \text { if } d_{G}(u) \leq 6 \\ n_{G}^{5}(u) \leq 1 & \text { if } d_{G}(u)=7 \\ n_{G}^{4^{-}}(u) \leq d_{G}(u)-7 & \text { if } 8 \leq d_{G}(u) \leq 9 \\ n_{G}^{3-}(u) \leq d_{G}(u)-7 & \text { if } 10 \leq d_{G}(u) \leq 11 \\ n_{G}^{2^{-}}(u) \leq 5 & \text { if } d_{G}(u)=12\end{cases}
$$

Let $H=G-V_{2^{-}}(G)$. Then, $d_{H}(u)=d_{G}(u)-n_{G}^{2^{-}}(u)$ for each $u \in V(H)$.

Claim 9. If $u \in V(H)$, then,

(1) $d_{H}(u) \geq 3$,

(2) $d_{H}(u)=d_{G}(u)$ if $3 \leq d_{G}(u) \leq 7$,

(3) $d_{H}(u) \geq 7$ if $8 \leq d_{G}(u) \leq 12$,

(4) $n_{H}^{5^{-}}(u)=0$ if $d_{H}(u)=7$ and $8 \leq d_{G}(u) \leq 12$,

(5) $n_{H}^{5^{-}}(u)=0$ if $d_{H}(u) \leq 6$.

Proof. Note that $3 \leq d_{G}(u) \leq 12$ by the definition of $H$ and Claim 2 for every $u \in V(H)$. By the definition of $H,(1),(2),(3)$, and (4) follow from Equation (1). Below, we prove (5).

(5) Suppose that $n_{H}^{5^{-}}(u) \geq 1$ when $d_{H}(u) \leq 6$. Without loss of generosity, set $d_{H}(u)=6$. Then, $d_{G}(u)=6$ by (2). Thus, $n_{G}^{5^{-}}(u)=0$ by Claim 3 , and $u$ has at least one $7^{+}$-neighbor $v$ in $G$ with $d_{H}(v) \leq 5$ by (2). Hence, $d_{H}(v)=d_{G}(v)-n_{G}^{2^{-}}(v) \geq 7$ by (3) and the definition of $H$, which is a contradiction. Therefore, the statement (5) holds.

By a similar discussion to that of Claim 9, we can obtain the following Claims 10 12.

Claim 10. If $d_{H}(u)=7$, then $n_{H}^{5}(u) \leq 1$.

Claim 11. If $d_{H}(u)=\ell$ with $8 \leq \ell \leq 9$, then,

(1) $n_{H}^{4^{-}}(u) \leq \ell-7$,

(2) $n_{H}^{5^{-}}(u) \leq \ell-7$ when $n_{H}^{4^{-}}(u) \geq 1$.

Claim 12. If $d_{H}(u)=\ell$ with $10 \leq \ell \leq 11$, then,

(1) $n_{H}^{3^{-}}(u) \leq \ell-7$,

(2) $n_{H}^{4^{-}}(u) \leq \ell-7$ when $n_{H}^{3^{-}}(u) \geq 1$,

(3) $n_{H}^{5}(u)=0$ when $n_{H}^{3^{-}}(u) \geq 1$ and $n_{H}^{4^{-}}(u)=\ell-7$.

By Claims 8 and 9, the below claim is immediate.

Claim 13. Let $C_{3}=v_{1} v_{2} v_{3} v_{1}$ be a 3-cycle in $H$. Then,

(1) $\left(d_{H}\left(v_{1}\right), d_{H}\left(v_{2}\right), d_{H}\left(v_{3}\right)\right) \neq\left(6^{-}, 6^{-}, 6^{-}\right)$,

(2) $n_{H}^{5^{-}}\left(v_{3}\right)=0$ when $\left(d_{H}\left(v_{1}\right), d_{H}\left(v_{2}\right), d_{H}\left(v_{3}\right)\right)=(6,6,7)$.

From now on, we always assume that $H$ has been embedded on the plane such that the number of crossings is as small as possible. Let $H^{\times}$be the associated planar graph of $H$ and $u v \in E\left(H^{\times}\right)$. If $u v$ is incident with two 3-faces and $u$ is a real $\ell$-vertex in $H^{\times}$, we call $u$ a bad $\ell$-neighbor of $v$. If $u v$ is incident with exactly one 3 -face and $u$ is a real 3-vertex in $H^{\times}$, we call $u$ a special 3-neighbor of $v$. Let $n_{H^{\times}}^{\ell b}(v)\left(n_{H^{\times}}^{3 s}(v)\right)$ denote the number of bad $\ell$-neighbors (special 3-neighbors) of $v$ in $H^{\times}$. Unless stated otherwise, an $\ell$-vertex 
represents a real $\ell$-vertex in the following. A 4 -face is called a good 4 -face if it is incident at most a real $5^{-}$-vertex and a bad 4-face otherwise. By Claims 1 and 9, the below Claims 14 and 15 are immediate.

Claim 14. If $d_{H}(u)=12$, then,

(1) $n_{H^{\times}}^{3 b}(u) \leq 4, n_{H^{\times}}^{3 s}(u) \leq 6, n_{H^{\times}}^{4 b}(u) \leq 6$ and $n_{H^{\times}}^{5 b}(u) \leq 6$,

(2) $n_{H^{\times}}^{3 s}(u)+n_{H^{\times}}^{4 b}(u)+n_{H^{\times}}^{5 b}(u)=0$ when $n_{H^{\times}}^{3 b}(u)=4$.

Claim 15. Every $k$-face is incident with at most $\left\lfloor\frac{k}{2}\right\rfloor$ real $5^{-}$-vertices in $H^{\times}$.

Let $\gamma\left(H^{\times}\right)$denote the number of connected components of $H^{\times}$. Note that each plane graph has exactly one unbounded face. Then, we have

$$
\sum_{f \in F\left(H^{\times}\right)} d_{H^{\times}}(f)=2\left|E\left(H^{\times}\right)\right|
$$

and Euler's formula

$$
\left|V\left(H^{\times}\right)\right|-\left|E\left(H^{\times}\right)\right|+\left|F\left(H^{\times}\right)\right|=1+\gamma\left(H^{\times}\right) .
$$

Below, we will apply the discharging method [13] on the planar graph $H^{\times}$to complete the proof of Theorem 3. Set $\omega(v)=d_{H^{\times}}(v)-6$ for any $v \in V\left(H^{\times}\right)$and $\omega(f)=2 d_{H^{\times}}(f)-$ 6 for any $f \in F\left(H^{\times}\right)$. By Euler's formula,

$$
\left|V\left(H^{\times}\right)\right|-\left|E\left(H^{\times}\right)\right|+\left|F\left(H^{\times}\right)\right|=1+\gamma\left(H^{\times}\right)
$$

and

$$
\sum_{v \in V\left(H^{\times}\right)} d_{H^{\times}}(v)=\sum_{f \in F\left(H^{\times}\right)} d_{H^{\times}}(f)=2\left|E\left(H^{\times}\right)\right| .
$$

One can obtain

$$
\sum_{v \in V\left(H^{\times}\right)}\left(d_{H^{\times}}(v)-6\right)+\sum_{f \in F\left(H^{\times}\right)}\left(2 d_{H^{\times}}(f)-6\right)=-6\left(1+\gamma\left(H^{\times}\right)\right) .
$$

In order to redistribute charges on $T\left(H^{\times}\right)$and keep the total charges unchanged, we make some discharging rules as follows.

(R1) Let $u v$ be an edge of $H^{\times}$.

(R1.1) If $u$ is a bad 3-neighbor of $v, u$ receives 1 from $v$.

(R1.2) If $u$ is a special 3-neighbor of $v, u$ receives $\frac{1}{2}$ from $v$.

(R1.3) If $u$ is a bad 4-neighbor of $v, u$ receives $\frac{1}{2}$ from $v$.

(R1.4) If $u$ is a bad 5-neighbor of $v, u$ receives $\frac{1}{5}$ from $v$.

(R2) Each real $\ell$-vertex with $3 \leq \ell \leq 5$ receives 1 from every $4^{+}$-face incident with it.

(R3) Let $u v$ be an edge and $u$ be a false 4 -vertex of $H^{\times}$.

(R3.1) If $d_{H^{\times}}(v)=7, u$ receives 1 when $v$ is not adjacent to any real $5^{-}$-vertex and $\frac{4}{5}$ otherwise from $v$.

(R3.2) If $d_{H^{\times}}(v)=\ell$ with $8 \leq \ell \leq 12, u$ receives 2 when $n_{H^{\times}}^{3}(v)<n_{H}^{3}(v)$ and 1 otherwise from $v$.

(R3.3) If $f$ is a good 4-face or $5^{+}$-face incident with $u, u$ receives 1 from $f$.

For each $z \in V\left(H^{\times}\right) \cup F\left(H^{\times}\right)$, we use $\omega^{\prime}(z)$ to represent the new charge after applying the rules. Then, one can obtain

$\sum_{z \in V\left(H^{\times}\right) \cup F\left(H^{\times}\right)} \omega^{\prime}(z)=\sum_{v \in V\left(H^{\times}\right)}\left(d_{H^{\times}}(v)-6\right)+\sum_{f \in F\left(H^{\times}\right)}\left(2 d_{H^{\times}}(f)-6\right)=-6\left(1+\gamma\left(H^{\times}\right)\right)<0$.

Thus, there exists a vertex or a face whose charge is negative. 
Firstly, it is easy to verify that the new charge of every face is non-negative by Claim 15 and the discharging rules.

Next, we show that the new charge of every real vertex is non-negative. Pick arbitrarily a real vertex $v$ from $V(H)$. Note that $v$ is adjacent to at most a false 4-vertex as $G$ (and thus, $H)$ is an IC-planar graph. Note also that $3 \leq d_{H}(v)=d_{H^{\times}}(v) \leq 12$ and $n_{H^{\times}}^{3}(v) \leq n_{H}^{3}(v)$ by the definition of $H^{\times}$and Claim 9.

(1) For $v \in V_{3}(H)$. If $v$ is incident with three $4^{+}$-faces, then $\omega^{\prime}(v)=3-6+3 \times 1=0$ by (R2). If $v$ is incident with two $4^{+}$-faces, it is a common special 3-neighbor of two neighbors, and so $\omega^{\prime}(v)=3-6+2 \times 1+2 \times \frac{1}{2}=0$ by (R1.2) and (R2). If $v$ is incident with one $4^{+}$-face, then it is a special 3-neighbor of one neighbor and a common bad 3-neighbor of the other two neighbors, respectively, and so $\omega^{\prime}(v)=3-6+1 \times 1+1 \times 1+2 \times \frac{1}{2}=0$ by (R1.1), (R1.2), and (R2). If $v$ is not incident with any $4^{+}$-faces, it is a common bad 3-neighbor of three neighbors; so, $\omega^{\prime}(v)=3-6+3 \times 1=0$ by (R1.1).

(2) For $v \in V_{4}(H)$. If $v$ is incident with at least two $4^{+}$-faces, $\omega^{\prime}(v) \geq 4-6+2 \times 1=0$ by (R2). If $v$ is incident with one $4^{+}$-face, it is a common bad 4-neighbor of two neighbors; so, $\omega^{\prime}(v)=4-6+1 \times 1+2 \times \frac{1}{2}=0$ by (R1.3) and (R2). If $v$ is not incident with any $4^{+}$-faces, it is a common bad 4-neighbor of four neighbors; so, $\omega^{\prime}(v)=4-6+4 \times \frac{1}{2}=0$ by (R1.3).

(3) For $v \in V_{5}(H)$. If $v$ is incident with at least one $4^{+}$-faces, $\omega^{\prime}(v) \geq 5-6+1 \times 1=0$ by (R2). If $v$ is not incident with any $4^{+}$-faces, it is a common bad 5-neighbor of five neighbors; so, $\omega^{\prime}(v)=5-6+5 \times \frac{1}{5}=0$ by (R1.4).

(4) For $v \in V_{6}(H) . \omega^{\prime}(v)=6-6=0$ as no rule is applied to it.

(5) For $v \in V_{7}(H)$. Note that $v$ is adjacent to at most a real 5-vertex in $H$ (and so, in $H^{\times}$) by Claim 10. If $v$ is not adjacent to any real 5-vertex in $H^{\times}, \omega^{\prime}(v) \geq 7-6-1 \times 1=0$ by (R3.1). If $v$ is adjacent to a real 5-vertex in $H^{\times}, \omega^{\prime}(v) \geq 7-6-1 \times \frac{4}{5}-1 \times \frac{1}{5}=0$ by (R1.4) and (R3.1).

(6) For $v \in V_{\ell}(H)$ with $8 \leq \ell \leq 9$. If $n_{H^{\times}}^{3}(v)=n_{H}^{3}(v), \omega^{\prime}(v) \geq \ell-6-1 \times$ $1-\max \left\{(\ell-7) \times 1,\left\lfloor\frac{\ell}{2}\right\rfloor \times \frac{1}{5}\right\}=0$ by (R1), (R3.2), and Claim 11. If $n_{H^{\times}}^{3}(v)<n_{H}^{3}(v)$, $n_{H}^{3}(v) \geq 1$; so, $v$ is adjacent to at most $(\ell-8)$ real $5^{-}$-vertices in $H^{\times}$by Claim 11 and the definition of $H^{\times}$. Thus, $\omega^{\prime}(v) \geq \ell-6-1 \times 2-(\ell-8) \times 1=0$ by (R1) and (R3.2).

(7) For $v \in V_{\ell}(H)$ with $10 \leq \ell \leq 11$. If $n_{H^{\times}}^{3}(v)=n_{H}^{3}(v), \omega^{\prime}(v) \geq \ell-6-1 \times$ $1-\max \left\{(\ell-7) \times 1,\left\lfloor\frac{\ell}{2}\right\rfloor \times \frac{1}{2}\right\}=0$ by (R1), (R3.2), and Claim 12. If $n_{H^{\times}}^{3}(v)<n_{H}^{3}(v)$, $n_{H}^{3}(v) \geq 1$; so, $v$ is adjacent to at most $(\ell-8)$ real $4^{-}$-vertices in $H^{\times}$by Claim 12 and the definition of $H^{\times}$. Thus, $\omega^{\prime}(v) \geq \ell-6-1 \times 2-\max \left\{(\ell-8) \times 1,\left\lfloor\frac{\ell}{2}\right\rfloor \times \frac{1}{5}\right\}=0$ by (R1) and (R3.2).

(8) For $v \in V_{12}(H)$. By Claim $14, \omega^{\prime}(v) \geq 12-6-1 \times 2-\max \left\{4 \times 1,\left\lfloor\frac{\ell}{2}\right\rfloor \times \frac{1}{2}\right\}=0$ by (R1) and (R3.3).

Hence, the new charge of every real vertex is non-negative.

Finally, we prove the new charge of every false 4-vertex is non-negative. Choose arbitrarily a false 4-vertex $u$ from $V\left(H^{\times}\right) \backslash V(H)$. Note that $u$ is adjacent to at most two $5^{-}$-vertices in $H^{\times}$by the definition of $H^{\times}$and Claim 9. Assume that the edges $v_{1} v_{2}$ and $v_{3} v_{4}$ intersect at $u$ in $H$. Then, $N_{H^{\times}}(u)=\left\{v_{1}, v_{2}, v_{3}, v_{4}\right\}$. The subgraph of $H^{\times}$induced by $N_{H^{\times}}(u) \cup\{u\}$ is denoted by $H^{\times}\left[N_{H^{\times}}(u) \cup\{u\}\right]$. Note that if $v_{i}\left(v_{i+2}\right)$ is a $5^{-}$-vertex with $i=1,2, v_{i+2}\left(v_{i}\right)$ is a $7^{+}$-vertex and $v_{i+2}\left(v_{i}\right)$ is not adjacent to any real $5^{-}$-vertex when $v_{i+2}\left(v_{i}\right)$ is a 7-vertex in $H^{\times}$by the definition of $H^{\times}$and Claim 9.

(9) Let $H^{\times}\left[N_{H^{\times}}(u) \cup\{u\}\right]$ contain no 3-face (see Figure 3). Then, $u$ is incident with at least three faces, each of which either is a good 4 -face or a $5^{+}$-face since $n_{H^{\times}}^{5^{-}}(u) \leq 2$ by Claim 9; so, $\omega^{\prime}(u) \geq 4-6+3 \times 1=1$ by (R3.3).

(10) Let $H^{\times}\left[N_{H^{\times}}(u) \cup\{u\}\right]$ contain exactly one 3-face (see Figure 4). Note that $n_{H^{\times}}^{5^{-}}(u) \leq 2$ and at most one of $d_{H^{\times}}\left(v_{1}\right)$ and $d_{H^{\times}}\left(v_{2}\right)$ is equal to 3 by Claim 9. If $d_{H^{\times}}\left(v_{1}\right) \neq 3$ and $d_{H^{\times}}\left(v_{2}\right) \neq 3, \omega^{\prime}(u) \geq 4-6+2 \times 1=0$ by (R3.3) since $u$ is incident with at least two faces, each of which either is a good 4 -face or a $5^{+}$-face. If $d_{H^{\times}}\left(v_{1}\right)$ is equal to $3, \omega^{\prime}(u) \geq 4-6+2 \times 1+1 \times 1-1 \times \frac{1}{2}=\frac{1}{2}$ by (R1.2) and (R3) since $u$ is incident 
with at least two faces, each of which either is a good 4-face or a $5^{+}$-face. By symmetry, $\omega^{\prime}(u) \geq \frac{1}{2}$ when $d_{H^{\times}}\left(v_{2}\right)$ is equal to 3 .

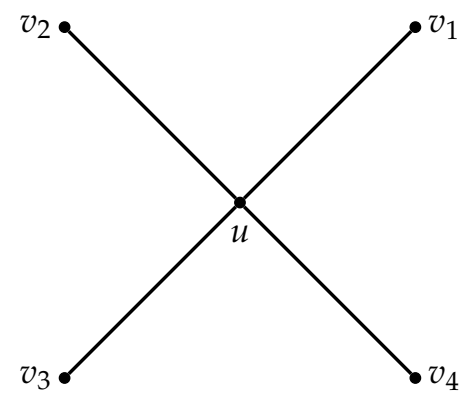

Figure 3. $H^{\times}\left[\{u\} \cup N_{H^{\times}}(u)\right]$ contains no 3-face.

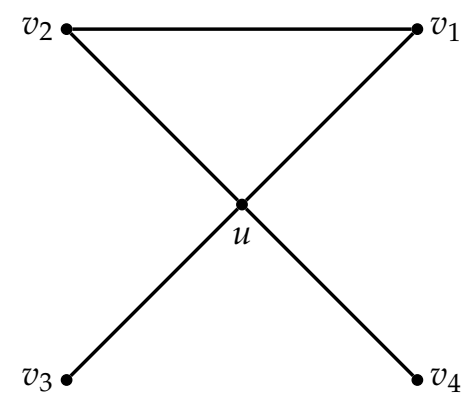

Figure 4. $H^{\times}\left[\{u\} \cup N_{H^{\times}}(u)\right]$ contains one 3-face.

(11) Let $H^{\times}\left[N_{H^{\times}}(u) \cup\{u\}\right]$ contain two 3-faces (see Figure 5). Note that $n_{H^{\times}}^{5^{-}}(u) \leq 2$ by Claim 9 and $v_{1}$ of (b) in Figure 5 is not a 3-vertex by Lemma 1. If $n_{H^{\times}}^{5^{-}}(u)=0, \omega^{\prime}(u) \geq$ $4-6+2 \times 1=0$ by (R3.3) since $u$ is incident with two faces, each of which either is a good 4 -face or a $5^{+}$-face. If $n_{H^{\times}}^{5^{-}}(u)=1, \omega^{\prime}(u) \geq 4-6+2 \times 1+1 \times 1-1 \times \frac{1}{2}=\frac{1}{2}$ by (R1.2) and (R3.3), since $u$ is incident with two faces, each of which either is a good 4 -face or a $5^{+}$-face. If $n_{H^{\times}}^{5^{-}}(u)=2, \omega^{\prime}(u) \geq 4-6+1 \times 1+2 \times 1-2 \times \frac{1}{2}=0$ by (R1.2) and (R3.3) since $u$ is incident with one face, which either is a good 4 -face or a $5^{+}$-face.

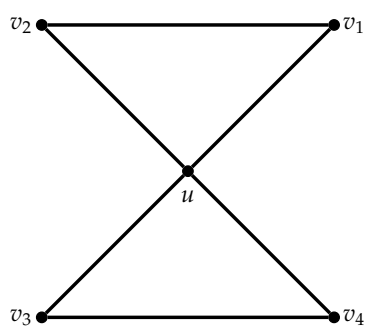

(a)

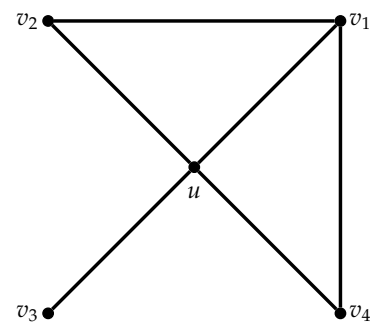

(b)

Figure 5. $H^{\times}\left[\{u\} \cup N_{H^{\times}}(u)\right]$ contains two 3-faces; (a) two nonadjacent 3-faces; (b) two adjacent 3-faces.

(12) Let $H^{\times}\left[N_{H^{\times}}(u) \cup\{u\}\right]$ contain three 3-faces (see Figure 6). Note that $n_{H^{\times}}^{5^{-}}(u) \leq 2$ by Claim 9 and neither $v_{1}$ nor $v_{2}$ is a 3-vertex by Lemma 1 in Figure 6 . If $n_{H^{\times}}^{5^{-}}(u)=0$, at least one neighbor of $u$ is a $7^{+}$-vertex, and when one neighbor of $u$ is 7-vertex, it is not adjacent to any real $5^{-}$-vertex by Claim 13 ; so, $\omega^{\prime}(u) \geq 4-6+1 \times 1+1 \times 1=0$ by (R1) and (R3.3) since $u$ is incident with one face, which either is a good 4 -face or a $5^{+}$-face. If $n_{H^{\times}}^{5^{-}}(u)=1$ and $n_{H^{\times}}^{3}(u)=0, u$ is adjacent to at least two $7^{+}$-vertices; so, $\omega^{\prime}(u) \geq 4-6+1 \times 1+2 \times$ $\frac{4}{5}-1 \times \frac{1}{2}=\frac{1}{10}$ by (R1.3), (R1.4), and (R3.3) since $u$ is incident with one face, which either 
is a good 4-face or a $5^{+}$-face. If $n_{H^{\times}}^{5^{-}}(u)=1$ and $n_{H^{\times}}^{3}(u)=1, d_{H^{\times}}\left(v_{3}\right)=3$ or $d_{H^{\times}}\left(v_{4}\right)=3$. Since $v_{3}$ and $v_{4}$ are symmetrical in Figure 6, we can set $d_{H^{\times}}\left(v_{3}\right)=3$. Then, $d_{H^{\times}}\left(v_{1}\right) \geq 8$, $d_{H^{\times}}\left(v_{2}\right) \geq 8$, and $n_{H^{\times}}^{3}\left(v_{1}\right)<n_{H}^{3}\left(v_{1}\right)$; so, $\omega^{\prime}(u) \geq 4-6+1 \times 1+1 \times 2-1 \times \frac{1}{2}=\frac{1}{2}$ by (R1.2), (R3.2), and (R3.3) since $u$ is incident with one face, which either is a good 4 -face or a $5^{+}$-face. If $n_{H^{\times}}^{5^{-}}(u)=2, d_{H^{\times}}\left(v_{3}\right) \leq 5, d_{H^{\times}}\left(v_{4}\right) \leq 5, d_{H^{\times}}\left(v_{1}\right) \geq 8$, and $d_{H^{\times}}\left(v_{2}\right) \geq 8$ by Claims 9 and 10. If $n_{H^{\times}}^{3}(u)=0, \omega^{\prime}(u) \geq 4-6+2 \times 1=0$ by (R3.2). If $n_{H^{\times}}^{3}(u)=1$, then we may as well set $d_{H^{\times}}\left(v_{3}\right)=3$ since $v_{3}$ and $v_{4}$ are symmetrical in Figure 6. Thus, $n_{H^{\times}}^{3}\left(v_{1}\right)<n_{H}^{3}\left(v_{1}\right)$; so, $\omega^{\prime}(u) \geq 4-6+1 \times 1+1 \times 2-1 \times \frac{1}{2}=\frac{1}{2}$ by (R1.2) and (R3.2). If $n_{H^{\times}}^{3}(u)=2, d_{H^{\times}}\left(v_{3}\right)=d_{H^{\times}}\left(v_{4}\right)=3$. Thus, $n_{H^{\times}}^{3}\left(v_{1}\right)<n_{H}^{3}\left(v_{1}\right)$ and $n_{H^{\times}}^{3}\left(v_{2}\right)<n_{H}^{3}\left(v_{2}\right)$. So, $\omega^{\prime}(u) \geq 4-6+2 \times 2-2 \times \frac{1}{2}=1$ by (R1.2) and (R3.2).

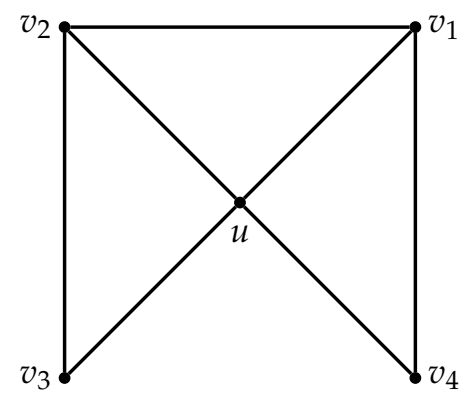

Figure 6. $H^{\times}\left[\{u\} \cup N_{H^{\times}}(u)\right]$ contains three 3-faces.

(13) Let $H^{\times}\left[N_{H^{\times}}(u) \cup\{u\}\right]$ contain four 3-faces (see Figure 7). Then, $d_{H^{\times}}\left(v_{i}\right) \neq 3$ for $i=1,2,3,4$ by Lemma 1 . Note that $n_{H^{\times}}^{5^{-}}(u) \leq 1$ by Claim 9. If $n_{H^{\times}}^{5^{-}}(u)=0$ and $n_{H^{+}}^{8^{+}}(u)=0$, then $u$ is adjacent to at least two 7-vertices by Claim 13. When $u$ is adjacent to two 7-vertices, each of the two 7-vertices is not adjacent to any real $5^{-}$-vertex by Claim 13 , and so $\omega^{\prime}(u) \geq 4-6+2 \times 1=0$ by (R3.1). When $u$ is adjacent to at least three 7-vertices, $\omega^{\prime}(u) \geq 4-6+3 \times \frac{4}{5}=\frac{2}{5}$ by (R3.1). If $n_{H^{\times}}^{5^{-}}(u)=0$ and $n_{H^{\times}}^{8^{+}}(u)=1$, we may as well set $d_{H^{\times}}\left(v_{1}\right) \geq 8$ by symmetry. Then, at least one of $v_{2}, v_{3}$ and $v_{4}$ is a 7 -vertex by Claim 13 . When one of $v_{2}, v_{3}$ and $v_{4}$ is a 7-vertex, this 7-vertex is not adjacent to any real $5^{-}$-vertex by Claim 13, and so $\omega^{\prime}(u) \geq 4-6+1 \times 1+1 \times 1=0$ by (R3.1) and (R3.2). When at least two of $v_{2}, v_{3}$ and $v_{4}$ are 7-vertices, $\omega^{\prime}(u) \geq 4-6+2 \times \frac{4}{5}+1 \times 1=\frac{3}{5}$ by (R3.1) and (R3.2). If $n_{H^{\times}}^{5^{-}}(u)=0$ and $n_{H^{\times}}^{8^{+}}(u) \geq 2, \omega^{\prime}(u) \geq 4-6+2 \times 1=0$ by (R3.2). If $n_{H^{\times}}^{5^{-}}(u)=1$ and $n_{H^{\times}}^{4}(u)=1, u$ is adjacent to three $8^{+}$-vertices by Claim 9; so, $\omega^{\prime}(u) \geq 4-6+3 \times 1-\frac{1}{2}=\frac{1}{2}$ by (R1.3) and (R3.2). If $n_{H^{\times}}^{5^{-}}(u)=1$ and $n_{H^{\times}}^{4}(u)=0, u$ is adjacent to three $7^{+}$-vertices by Claim 9; so, $\omega^{\prime}(u) \geq 4-6+3 \times \frac{4}{5}-\frac{1}{5}=\frac{1}{5}$ by (R1.4), (R3.1), and (R3.2).

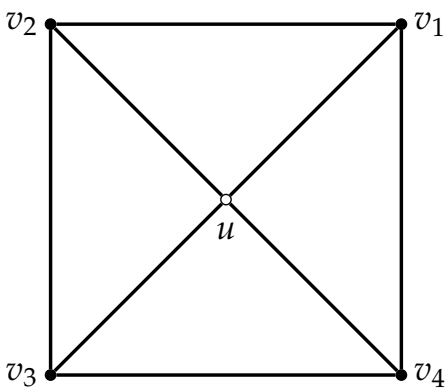

Figure 7. $H^{\times}\left[\{u\} \cup N_{H^{\times}}(u)\right]$ contains four 3-faces.

Hence, the new charge of every false 4-vertex is non-negative.

By the above analysis, we obtain that the new charge of every member in $V\left(H^{\times}\right) \cup$ $F\left(H^{\times}\right)$is non-negative. This is a contradiction. The proof of Theorem 3 is completed. 


\section{Conclusions}

In this paper, we proved that any IC-planar graph with $\Delta \geq 12$ satisfies the tnsd index conjecture by the discharging method and Combinatorial Nullstellensatz. Whether the bound $\Delta \geq 12$ can be improved is still open for future work. The difficulty in improving this bound is how to reduce the number of bad 3-neighbors of one vertex. Therefore, finding suitable technology to reduce the number of bad 3-neighbors of one vertex is our main future work in the study of the tnsd index of IC-planar graph.

Author Contributions: Methodology, D.Z.; software, F.C.; formal analysis, D.Z.; writing-original draft preparation, D.Z.; writing-review and editing, C.L. All authors have read and agreed to the published version of the manuscript.

Funding: This research was funded by Shangluo Science and Technology Plan Project (No. SK2017-40).

Institutional Review Board Statement: Not applicable.

Informed Consent Statement: Not applicable.

Data Availability Statement: Not applicable.

Acknowledgments: The authors are grateful to all anonymous reviewers and the editor for their inspiring and constructive comments and suggestions.

Conflicts of Interest: The authors declare no conflict of interest.

\section{References}

1. Pilśniak, M.; Woźniak, M. On the total-neighbor-distinguishing index by sums. Graphs Comb. 2015, 31, 771-782. [CrossRef]

2. Xu, C.; Ge, S.; Li, J. Neighbor sum distinguishing total chromatic number of 2-degenerate graphs. Discret. Appl. Math. 2018, 251, 349-352. [CrossRef]

3. Li, H.; Liu, B.; Wang, G. Neighbor sum distinguishing total coloring of $K_{4}$-minor-free graphs. Front. Math. China 2013, 8 , 1351-1366. [CrossRef]

4. Yang, D.; Sun, L.; Yu, X.; Wu, J.; Zhou, S. Neighbor sum distinguishing total chromatic number of planar graphs with maximum degree 10. Appl. Math. Comput. 2017, 314, 456-468. [CrossRef]

5. Wang, J.; Ma, Q.; Han, X. Neighbor sum distinguishing total colorings of triangle free planar graphs. Acta Math. Sin. (Engl. Ser.) 2015, 2, 216-224. [CrossRef]

6. Albertson, M.O. Chromatic number, independent ratio, and crossing number. Ars Math. Contemp. 2008, 1, 1-6. [CrossRef]

7. Song, W.; Duan, Y.; Miao, L. Neighbor Sum Distinguishing Total Coloring of Triangle Free IC-planar Graphs. Acta Math. Sin. (Engl. Ser.) 2020, 36, 292-304. [CrossRef]

8. Song, W.; Duan, Y.; Miao, L. Neighbor sum distinguishing total coloring of IC-planar graphs. Discret. Math. 2020, $343,111918$. [CrossRef]

9. Song, C.; Xu, C. Neighbor sum distinguishing total colorings of IC-planar graphs with maximum degree 13. J. Comb. Optim. 2020, 39, 293-303. [CrossRef]

10. Zhang, D. Neighbor Sum Distinguishing Total Choosability of IC-Planar Graphs without Theta Graphs $\Theta_{2,1,2}$. Mathematics 2021, 9, 708. [CrossRef]

11. Alon, N. Combinatorial nullstellensatz. Combin. Probab. Comput. 1999, 8, 7-29. [CrossRef]

12. Sun, L.; Yu, G.; Li, X. Neighbor sum distinguishing total choosability of 1-planar graphs with maximum degree at least 24 . Discrete Math. 2021, 344, 112190. [CrossRef]

13. Daniel, W.; Douglas, B. An introduction to the discharging method via graph coloring. Discrete Math. 2017, 340, 766-793. 